In the Case Report by Carla Vignaroli, Laura Di Sante, Paola Stano, Pietro E Varaldo \& Alessandro Camporese, titled 'Recurrent skin infection associated with nasal carriage of Panton-Valentine leukocidin-positive methicillin-susceptible Staphylococcus aureus closely related to the EMRSA-15 clone', which appeared in the January 2016 issue of Future Microbiology (11[1], 17-21 [2016]), the name of the author Laura Di Sante was presented incorrectly as:

\title{
Sante LD
}

The name has been corrected to:

\section{Di Sante L}

The editors of Future Microbiology would like to sincerely apologize for any inconvenience or confusion this may have caused our readers. 